\title{
Denosing CT/MRI Images Restoration using Radial Basis Function Neural Network
}

\author{
B.Baron Sam, A. Lenin Fred
}

\begin{abstract}
Medical imaging technology is becoming an important component of large numbers of applications such as diagnosis, treatment, survey and medical examination. Image restoration manages conveying back the bended image to its original domain. It re-establishes the corrupted image into keener image. This paper centers around evacuation of noise strategies in medical images with denoising a point by point overview has been completed on various image denoising methods and their exhibitions were evaluated and it is an activity to examine and evaluate various variations of denoising methods to enhance their execution and visual standard.
\end{abstract}

\section{INTRODUCTION}

Image Processing is a strategy to play out some operations on an images, retaining in thoughts that our cease intention is to get an advanced images or to do away with a few helpful facts from it. It is a type of signal processing in which input is an image and yield might be image or attributes related with that image. These days, image processing is among quick growing improvements. It frames centre studies territory inner designing and software program engineering disciplines as well.

Image processing basically carries the accompanying 3 ranges

- Importing the image by means of image procurement apparatus

- Evaluating and controlling the image

- Output wherein result can be adjusted image or report that depends on picture examination.

There are two styles of strategies applied for image processing to be specific i.E. Analog and Digital picture processing. Analog picture processing may be applied for the published papers like printouts and images. Image examiners utilize special fundamentals of translation while using those methods. The Digital image processing Strategies help on top of things of the advanced pictures via using computers. The pre-processing, improve and display are the 3 preferred levels that a extensive range of information want to experience at the same time as using virtual picture processing techniques.

\subsection{Artificial Neural Networks (Ann)}

ANN are group of models which are motivated by biological neural network (example: brain) and they are utilised to calculate the absolute values that can rely upon huge number of inputs which are unknown. Artificial neural networks are generally introduced as framework of interconnected neurons which trade messages between each

Revised Manuscript Received on April 12, 2019.

B.Baron Sam, Research Scholar, Faculty of Computing, Sathyabama Institute of Science and Technology, Chennai. T.N, India.

Dr. A. Lenin Fred, Principal, Mar Ephream College of Engineering \& Technology, Kanyakumari. T.N, India. other. These associations have numeric values that can be based on understanding, making neural network adaptive to input.

\subsection{Image Restoration}

Image Restoration is the process of restoring the original image from the corrupted image. The corrupted image is the output of familiar or unfamiliar degradation. Therefore image restoration may be characterised as a process of enhancing a sharp photograph from a corrupted image that's darkened with the aid of the degradation function. The procedure of image recuperation includes two sub bureaucracy. the primary process manages degradation nature of the image via calculating the blur and noise to the image and the opposite shape manages expelling noise from the corrupted image and we will restore the original image

\subsection{Degradation Model}

The original image is degraded by utilizing the degradation function and the added noise with the end goal that the corrupted image is presented in mathematical explanation:

$$
H(m, n)=G^{*} E(m, n)+F(m, n)
$$

In this equation, $H(m, n)$ is the processed image, $G$ is degradation function, $E(m, n)$ is original image and $F(m, n)$ is an Added noise

\section{RELATED RESEARCH WORK}

[1]Syed AmjadAli: They proposed a window based Multiwavelet transformation and thresholding. It evacuates the Additive Gaussian noise from the images and improves the quality of the CT images. The method comprises three sorts of processing which includes window based multiwavelet transformation and thresholding. The AWGN is completely evacuated in the third method along with improving the quality of the images with the help of filtering strategies. In this method a wavelet transform based method concentrates only on AWGN noise, where as other noises like Gaussian, salt and pepper etc. are not concentrated that much effectively.

[2]G.AmarTej : Here they have used pre-processing methods the neighbourhood pixels in the image to get new splendor estimations of the output image. These preprocessing methods and improvement in noise and resolution are the principle attributes in medicinal images. Noise is the primary variable that corrupts the vast majority of the medical imaging strategies. We have to preserve the edges and data from this noise. So we execute on the normal 
average filtering, median sifting. Wiener sifting and Wavelet denoising for removing noise and an addition based Discrete and stationary Wavelet Transform procedure for enhancing resolution. Pre-processing is possible by filters with high PSNR to high pass filter

[3]Chen Yang: Fundamental point is to enhance stomach area tumor low-measurements CT (LDCT) image quality by utilizing a two-stage technique patch wise nonlinear preparing is connected to expel the noise, that depends on a sparsity earlier in term of a scholarly dictionary An unsharp filtering method intends to upgrade the tissues and compensate the complexity brought on by the DL handling Results appear in this method is effective in filtering mottled noise and also enhancing tumor perceptibility. The entire calculation cost of the DL unsharp handling still should be quickened to meet the clinical necessity (under 0.5 second for every image). Second a few parameters are now set experimentally and need more investigations to approve their esteem.

[4]Keunho Park: This paper proposes a neural system based hybrid filter for evacuating quantum noise from the image. The proposed filter comprises of bilateral filters, a single or multiple neural edge enhancer, and a neural filter to consolidate them. The BF's consider the distinction in esteem from the neighbours, to save edges while smoothing. The NEE is utilized to unmistakably improve the edges from the images. The NF demonstrations like a fusion operator, and endeavours to develop an enhanced image. It focuses just on quantum noise.

[5]MojtabaFadaee: An ideal algorithm is displayed for de-noising medical images. It depends on enhanced form of nearby pixels gathering and principle part investigation. In neighbourhood pixels grouping algorithm, blocks matching method in view of L2 standard strategy. The proposed algorithm has de-noising and cleanup stages. First step gives an initial estimate of the image after de-noising. In the second step noises in the image which still remain are cleaned. So, Cleanup step removes the remained noises and causes the output de-noised images to have a more appropriate quality. Partial differential equations-based methods (e.g., nonlinear an isotropic diffusion method) for further improvements of medical image de-noising can be done

[6]Danni Ai: We propose adaptive tensor-based foremost part examination (AT-PCA) calculation for lowmeasurements CT image denoising. Pixels in the image are displayed by their close-by neighbours, and are demonstrated as a fix. Adaptive seeking wind computed to discover comparable fixes as preparing gatherings for further handling. He investigate the clinical images, the proposed AT-PCA strategy can stifle the noise, upgrade the edge, and enhance the image quality more adequately than NLM (Non nearby Mean)and KSVD (word reference learning calculation for making a lexicon for representations via a particular esteem deterioration approach denoising strategies. This paper depicted a novel image denoising structure to stifle the pixel noise of the low-dosage CT image, IE. During concealment that images are influenced

[7]David Boublil: In work this idea was represented for the instance of CT reproduction, finished with two fundamental calculations the FBP (Filter Back Projection) and the PWLS (Penalized Weighted Least Squares). We propose a regulated machine learning approach for boosting existing sign and image recuperation techniques. It depends on a nearby nonlinear combination of a few image estimation all got by applying a picked recreation calculation with various and the combination of the images is performed by feed forward neural system prepared on an arrangement of known illustrations. Numerical trials demonstrate a change in construction quality generally to existing immediate and iterative remaking techniques. Empiric results recommend that the nearby combination can enhance the determination and to include more visual nature of the CT images

[8]Campadelli.p: This paper we propose a completely programmed dark level based division structure that utilizes a quick walking technique Segmentation plan is general, and utilizes just settled and not basic anatomical information. Therefore, it can be effortlessly adjusted to independently fragment diverse stomach organs, by defeating issues because of the high entomb and intra quiet dark level and shape inconstancies, the extricated volumes are then joined to accomplish hearty outcomes. The framework execution has been assessed on the information of 40 patients, by contrasting the naturally distinguished organ volumes with the organ limits physically followed by three specialists. The great nature of the accomplished outcomes is demonstrated by the way that they are equivalent to the inter and intra individual changeability of the manual division delivered by specialists. Additionally preparing strategy can actualized with a specific end goal to envision the organs clearly

[9]Jiahui Wang: In division of lungs with extreme interstitial lung illness (ILD) in thoracic processed tomography $(\mathrm{CT})$ is a critical and troublesome errand in the advancement of PC supported finding (CAD) semi sections the lungs. We initially utilized a CT esteem thresholding strategy to get an underlying lung evaluate, including typical and mellow ILD lung parenchyma. We then utilized surface element images to additionally recognize irregular lung locales with serious ILD. At long last, we joined the distinguished abnormal lung districts with the underlying lungs to create the last lung division result. Our lung division strategy gave precise division results to strange CT checks with extreme ILD and would be valuable for creating CAD frameworks for evaluation, discovery, and conclusion of ILD. However the commotions introduce in the image ought to be evacuated

\section{PROPOSED SYSTEM}

In this section, it is explained the results of research and at the same time is given the comprehensive discussion. Results can be presented in figures, graphs, tables and others that make the reader understand easily [2], [5]. The discussion can be made in several sub-chapters. Degradation in the image is due to Blur due to misfocus, Image sensor noise, Blur due to motion and Noise from transmission channel. Hence we develop a robust and efficient soft computing based neural network restoration algorithm for 
CT, MR images. We use three filters namely adaptive filter, bilateral filter and RBFNN filter (Radial basis function neural network filter). Using this the image restoration filters the scan images are removed from noise and restoration is done at maximum possible level and we get high PSNR ratio

\subsection{Rbfnn Filter: Radial Basic Function Neural Network Filter Design}

For the most part, a RBFNN comprises of three layers: the Info layer, the RBF layer (shrouded layer) and the yield layer. The contributions of shrouded layer are to close blends of scalar weights and the info vector $\mathrm{xx} 1, \mathrm{x} 2, \mathrm{x} 3, \mathrm{x} 4, \mathrm{x} 5, \mathrm{x} 6 \ldots . .$, where the scalar weights are appointed solidarity values. In this manner the entire information vector appears to every neuron in the shrouded layer. The approaching vectors are mapping by the outspread premise works in each concealed hub. The yield layer yields a vector $\mathrm{y}, \mathrm{y} 1, \mathrm{y} 2, \mathrm{y} 3, \mathrm{y} 4, \mathrm{y} 5 \ldots .$. For $\mathrm{m}$ yields by direct mix of the yields of concealed hubs to deliver the last yield. Fig. 1

$$
y=f(x)=\sum_{i=1}^{h} w i(x)
$$

Where $f(x)$ is the last output, $h$ is the aggregate number of hidden nodes. An outspread premise capacity is a multidimensional capacity that depicts the separation between a given information vector a pre-characterized focus vector

The underlying focuses can be resolved, for instance, by $\mathrm{K}$-implies bunching of some of the info tests. Spread initial values $\sigma \mathrm{i}$ could be chosen as the average of the nearest neighbour distances among the initialized canters and trained later, or fixed during the training process, this parameter is generally fixed after multiple learning experimentations.

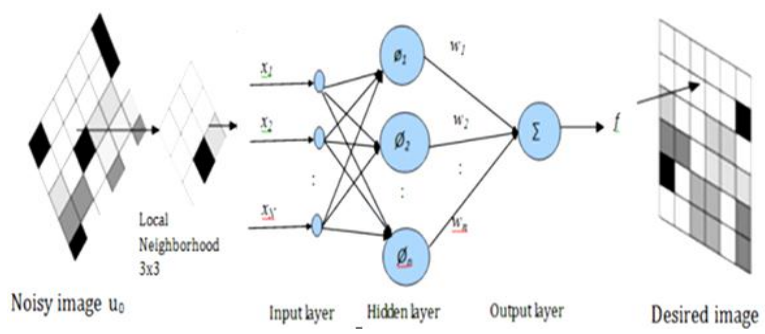

Figure 1. Radial Basic Function Neural Network Filter Design

\section{METHODOLOGIES}

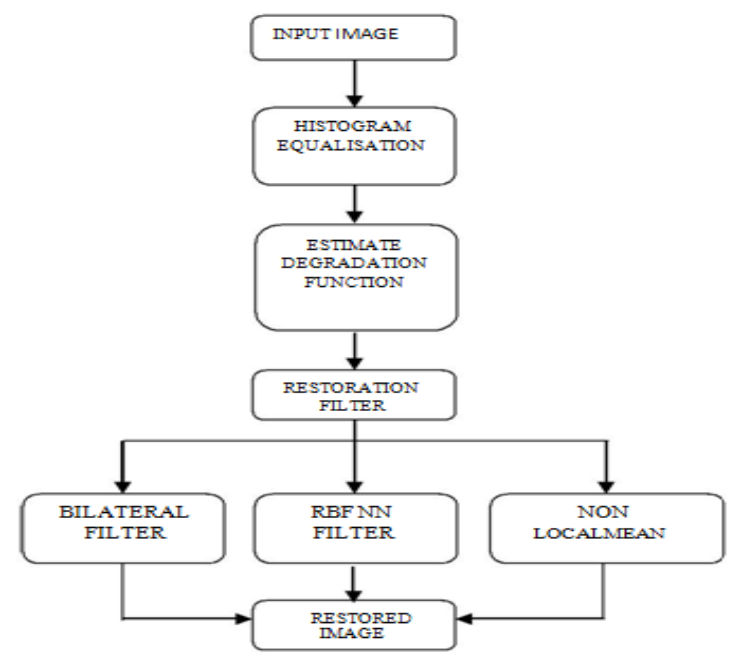

Figure 2 Flow diagram

\subsection{Input Image}

Mat lab can procedure pictures of layout such that they may be of (jpeg, bmp) codecs. Here, we take medical images as our input (i.e., CT and MRI scan images).Each pixel is a three-dimensional vector values with range $[0,255]$. These numbers are beneficial in figuring out the quantity of Red-Green-Blue (RGB).So a colour picture is certainly three gray scale images. When displaying a single channel, Mat lab likes to apply the pink-blue jet shade map but now we trade it to a grey scale shade map type: Colour map gray

\subsection{Adaptive Histogram Equalization}

An elective way to deal with histogram balance. We are performing translimited versatile histogram levelling (CLAHE) utilizing adapthistequalization work While histogram balance deals with the whole picture, adapthistequalization chips away at Small locales in the picture called tiles.. In the wake of playing out the evening out, adapthistequalization joins neighbouring tiles utilizing bilinear introduction strategy to dispense with misleadingly incited limits. Adapthistequalization delivers a yield picture having values similarly passed on all through the degree.

\subsection{Degradation Function}

Image recuperation algorithms are intended to abuse characteristic of a signal and degradation, accurate data of the corruption is key to build up an effective image reclamation calculation. There are ways to deal with accumulate facts about degradation.

APPROACH 1. It is to gather facts from the degraded photo itself. Here, we are able to discover the regions inside the image $B(\mathrm{~m} 1, \mathrm{~m} 2)$. It is to accumulate facts for precise location of the images as $\mathrm{F}(\mathrm{m} 1, \mathrm{~m} 2)$ and the degraded picture the equal place as $\mathrm{G}(\mathrm{m} 1, \mathrm{~m} 2)$. Then factor is kind of related through

$\mathrm{G}\left(\mathrm{m}_{1}, \mathrm{~m}_{2}\right)=F\left(\mathrm{~m}_{1}, \mathrm{~m}_{2}\right) * B\left(\mathrm{~m}_{1}, \mathrm{~m}_{2}\right)$ 
Since $F(\mathrm{~m} 1, \mathrm{~m} 2)$ and $\mathrm{G}(\mathrm{m} 1, \mathrm{~m} 2)$ is assumed known $\mathrm{b}(\mathrm{m} 1, \mathrm{~m} 2)$ can be claculated from (4.3.1) $\quad g(a, b)$ as

$$
g(a, b) \frac{1}{T} \iint_{t}^{T / 2}=-T / 2 f\left(a-a_{0}(\mathrm{t}), \mathrm{b}-\mathrm{b}_{0}(\mathrm{t})\right) \mathrm{dt}
$$

Where $\mathrm{a} 0(\mathrm{t})$ and $\mathrm{b} 0(\mathrm{t})$ speak to the level and vertical interpretations of $f(a, b)$ at time $t$ with respect to the imaging framework, and $\mathrm{T}$ is the presentation term. The Fourier change area, (4.3.2) can be characterized as

$$
\mathrm{g}\left(\Omega_{\mathrm{a},}, \Omega_{\mathrm{b}}\right)=\int_{\mathrm{x}}^{\infty}=-\infty \int_{\mathrm{y}}^{\infty}=-\infty \mathrm{g}(\mathrm{a}, \mathrm{b}) \mathrm{e}^{-\mathrm{j}} \mathrm{e}^{-\mathrm{j}} \Omega_{\mathrm{a},} \mathrm{a} e^{-j} \Omega_{\mathrm{b}} \mathrm{bdadb}
$$

Where $\mathrm{g}\left(\Omega_{\mathrm{a}}, \Omega_{\mathrm{b}}\right)$ is the Fourier change of $\mathrm{g}(\mathrm{a}, \mathrm{b})$.Simplify (5.3.3), we got

$$
\mathrm{g}\left(\Omega_{\mathrm{a},} \Omega_{\mathrm{b}}\right)=f\left(\Omega_{\mathrm{x}}, \Omega_{\mathrm{b}}\right) \mathrm{b}\left(\Omega_{\mathrm{a},} \Omega_{\mathrm{b}}\right)
$$

From (4.3.4), obviously the planar development darken can be viewed as convolution of $f(a, b)$ with $b(a, b)$ whose the Fourier change $\mathrm{B}\left(\Omega_{\mathrm{a}}, \Omega_{\mathrm{b}}\right)$

$$
\mathrm{b}(\Omega a, \Omega b)=\frac{1}{T} \int_{-T / 2}^{T / 2} e^{-\mathrm{j}} \Omega_{a,} \mathrm{a}_{0}(\mathrm{t}) \mathrm{e}^{-\mathrm{j}} \Omega_{b} \mathrm{~b}_{0}(\mathrm{t}) \mathrm{dt}
$$

is characterized by $(4.3 .4 b)$. The capacity $b(a, b)$ is once in a while alluded to as the obscuring capacity, since $b(a, b)$ commonly blur and low pass character of the image. It is in like manner implied as the point spread capacity, since it spreads an impulse. Exactly when there is no development, with the end goal that $\mathrm{a} 0(\mathrm{t})=0$ and $\mathrm{b} 0(\mathrm{t})=0, \mathrm{~B}(\omega \mathrm{a}, \omega \mathrm{b})$ is 1 . And $g(a, b)$ is $f(a, b)$.If there is straight movement along the ' $a$ ' heading so that $\mathrm{b}_{0}(\mathrm{t})=0$ and $\mathrm{a}_{0}(\mathrm{t})=\mathrm{kt}$ then $\mathrm{B}\left(\Omega_{\mathrm{a},}, \Omega_{\mathrm{b})}\right.$ in (5.3.4) reduces to

$$
\mathrm{B}\left(\left(\Omega_{\mathrm{a}}, \Omega_{\mathrm{b})}=\frac{\sin \frac{\Omega \mathrm{a} \mathrm{KT}}{2}}{\frac{\Omega \mathrm{a} \mathrm{KT}}{2}}\right.\right.
$$

A discrete image $\mathrm{G}(\mathrm{m} 1, \mathrm{~m} 2)$ might be roughly demonstrated

$$
\mathrm{G}\left(\mathrm{m}_{1 \mathrm{~m}} \mathrm{n}_{2}\right)=\mathrm{F}\left(\mathrm{m}_{1}, \mathrm{~m}_{2}\right) * \mathrm{~B}\left(\mathrm{~m}_{1}, \mathrm{~m}_{2}\right)
$$

Where $B(\omega 1, \omega 2)$ the discrete-space Fourier change of $\mathrm{B}(\mathrm{m} 1, \mathrm{~m} 2)$, is the associated variant of $\mathrm{B}(\omega \mathrm{a}, \omega \mathrm{b})$ in $(5.3 .4 \mathrm{~b})$. Distinctive models in which the debasement might be assessed from its framework join film grain uproar clouding in light of diffraction - obliged optics, and spot bustle.

\section{RESTORATION FILTERS}

\subsection{Improved Bilateral Filter}

The separate channel is a nonlinear direct technique in which it does spatial averaging without smoothing edges and it is a suitable picture denoising framework. It in like way can be related with the blocking old rarities lessening. A pressing issue with the utilization of the relative channel is the channel's confirmation parameters, which influence the results essentially. Another examination excitement of individual channel is growing speed of the tally speed. There are three standard duties of this suggestion. The essential responsibility here is sixth exploratory examination of the perfect corresponding direct parameter decision in picture denoising, where two-sided filtering is associated with the low-repeat sub band of a banner rotted using a Wavelet channel the second duty is that we acquaint a spatially versatile strategy with decline involving relics. The test outcomes display that the adaptable method can redesign the idea of restored images fundamentally better than that of the standard two-sided channel. The third duty exhibits a difference in the snappy complementary channel, to such a degree, that here, we use a mix of multi windows to evaluate the Gaussian channel all the more exactly

\subsection{Non-Local Means Method}

Efros and Leung were made the possibility of selfsimilarity for surface amalgamation. An instance of selfresemblance is appeared in Figure underneath. Most by far of the pixels in an unclear section from $p$ will have close neighborhoods to p's neighborhood. The self-similarity uncertainty can be mauled to denoise a image. Pixels With comparative neighborhoods can be utilized to focus the denoised estimation of a pixel.

Every pixel $\mathrm{p}$ of the non-nearby methods denoised picture is then assessed with the accompanying formulae

$$
\mathrm{NM}(\mathrm{L})(\mathrm{a})=\sum_{b: L} w(a, b) L(b)
$$

With the end goal that, here $\mathrm{L}$ is the picture that are noisy, weights are spoken to by $\mathrm{w}(\mathrm{p}, \mathrm{q})$ and it meet the accompanying conditions $0 \leq \mathrm{w}(\mathrm{a}, \mathrm{b}) \leq 1$ and $\sum_{b} w(a, b)=1$

To figure the closeness between two neighborhoods take the weighted squares contrasts whole between the two neighborhoods by formula.

$$
d(a, b)=\left(L: N_{a}\right) L\left(N_{b}\right) p^{2}, Q
$$

$\mathrm{F}$ is the neighbourhood filters which can be carried out to the squared distinction of the neighbourhoods and the weights are computed using the subsequent system:

$\mathrm{W}(\mathrm{a}, \mathrm{b})-\mathrm{z} 1(\mathrm{a}) \mathrm{e}-\mathrm{d}(\mathrm{a}, \mathrm{b}) \mathrm{z}(\mathrm{a})$ is the normalizing consistent which are defined as $\mathrm{z}(\mathrm{a})-\sum_{b}(\mathrm{e}){ }^{\mathrm{d}} \mathrm{N}^{(\mathrm{a}, \mathrm{b})}$ which $\mathrm{p}$ is that weight-decay manipulate parameter. As we referred to, $\mathrm{F}$ is the neighbourhood filter with radius that's precise as Rsim. The weights of $\mathrm{F}$ are calculated the usage of the subsequent formula:

$$
\frac{1}{R \operatorname{sim}}=\sum_{i=m}^{R \operatorname{sim}} \frac{1}{(2 * i+1)^{2}}
$$

Here, $m$ is the department the weights is from the factor of convergence of the channel. The channel offers extra weight to pixels near the factor of convergence of the sector, and much less weight to pixels close to the area's area. From above has an awesome situation when $b=a$. this is in mild of the fact that the load with the give up aim that w(a,a) may be much be greater essential than the weights from each different pixel. in the photo. by means of definition this looks desirable in light of the way that every zone resembles itself. To maintain pixel $\mathrm{p}$ from over-measuring itself let $\mathrm{w}$ $(\mathrm{a}, \mathrm{a})$ be proportionate to the most top notch weight of alternative pixels, or in greater logical terms

$$
\mathrm{W}(\mathrm{a}, \mathrm{a})=\max \{\mathrm{w}(\mathrm{a}, \mathrm{b}) \mid \mathrm{a} \neq \mathrm{b}\}
$$

\subsection{Rbfnn Filter (Radial Basis Function Neural Network)}

RBFNN comprises of 3 layers: the info layer, the RBF layer (concealed layer) and the yield layer. The contributions of shrouded layer are the straight blends 15 scalar Weights and the information vector, where the scalar 
weights are allotted solidarity values. The RBFNN has been utilized to the total harmonic content in power electronics converter wave forms based on the $\mathrm{p}$-q theory. The oddity of this strategy is the utilization of the RBFNN preparing system in view of a different and consecutive technique called the cross breed inclining process. In this strategy, the focuses of the RBFNN were chosen in view of the Kimplies grouping technique.

\section{QUALITY MEASURES}

\subsection{Mean Square Error [MSE]}

The average of the squares of the errors is measured by MSE. It calculates the difference between the estimator and what is estimated.

$$
\mathrm{MSE}=\frac{1}{M 1 N 1} \Sigma M 1_{x=1} \Sigma N 1_{\mathrm{Y}=1}|\mathrm{I}(\mathrm{x}, \mathrm{y})-\mathrm{I}(\mathrm{x}, \mathrm{y})|^{2}
$$

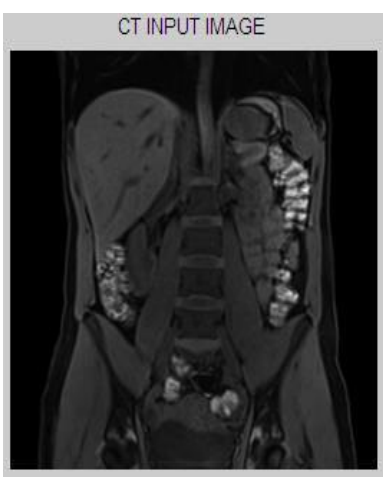

CT input Image

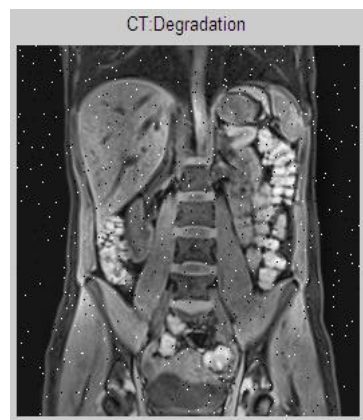

Noises in CT image

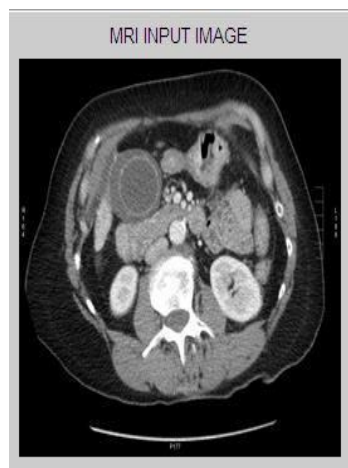

MRI input Image

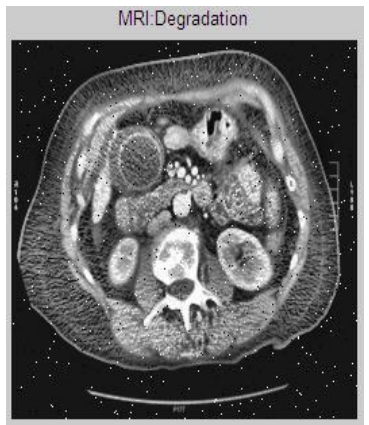

Noises in MRI image

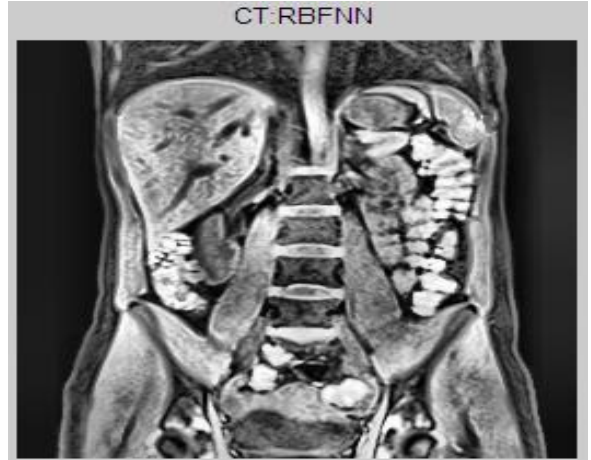

RBFNN filter in CT image
$\mathrm{I} \Sigma \mathrm{RM} 1 * \mathrm{M} 2$ is the clear image without any noise.

To determine the difference and similarity between the input and destined output image we use certain quality measures. It estimates the match between the images. Some of them are,

\subsection{Peak Signal To Noise Ratio}

The PSNR represents a measure of the peak error.

\section{PSNR = 10log10Nmax/ MSE}

The maximum fluctuation in the input image is represented by $\mathrm{N}$ max

\section{RESULTS}

The snap shot of the results given below and implemented in mat lab 2013

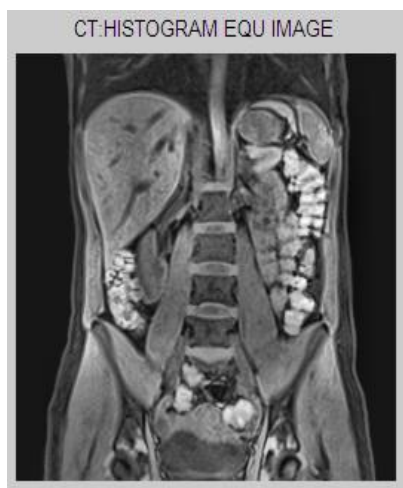

Histogram equalization in $\mathrm{CT}$ image

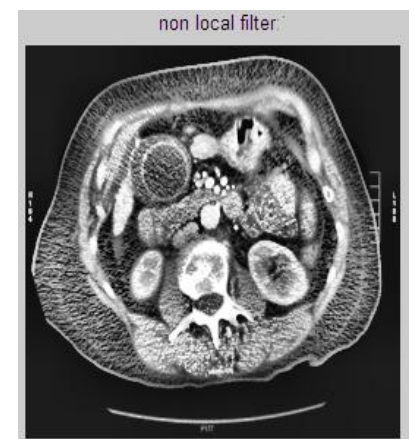

Non Local filter in CT image Bilateral filter in MRI image

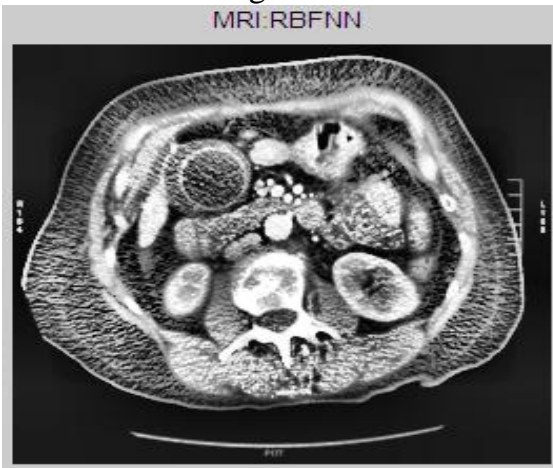

RBFNN filter in MRI image

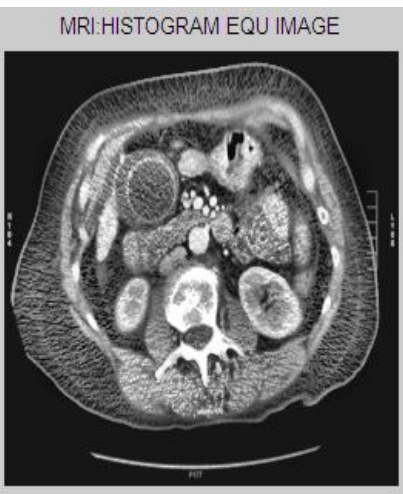

Histogram equalization in MRI image

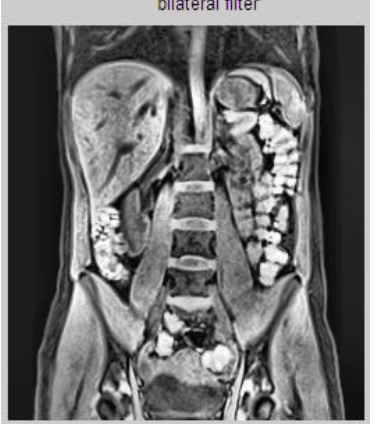

Figure 3 Results

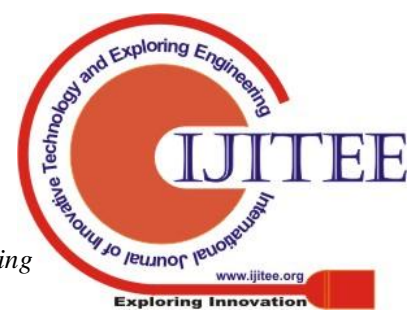


Table 1 Quality Measures

\begin{tabular}{cccc}
\hline Quality Measures & Non-Local Means & Improved Bilateral Filter & RBFNN Filter(Proposed) \\
\hline PSNR & 60.5226 & 68.9614 & 69.828 \\
MSE & 0.0577 & 0.0083 & 0.0068 \\
\hline
\end{tabular}
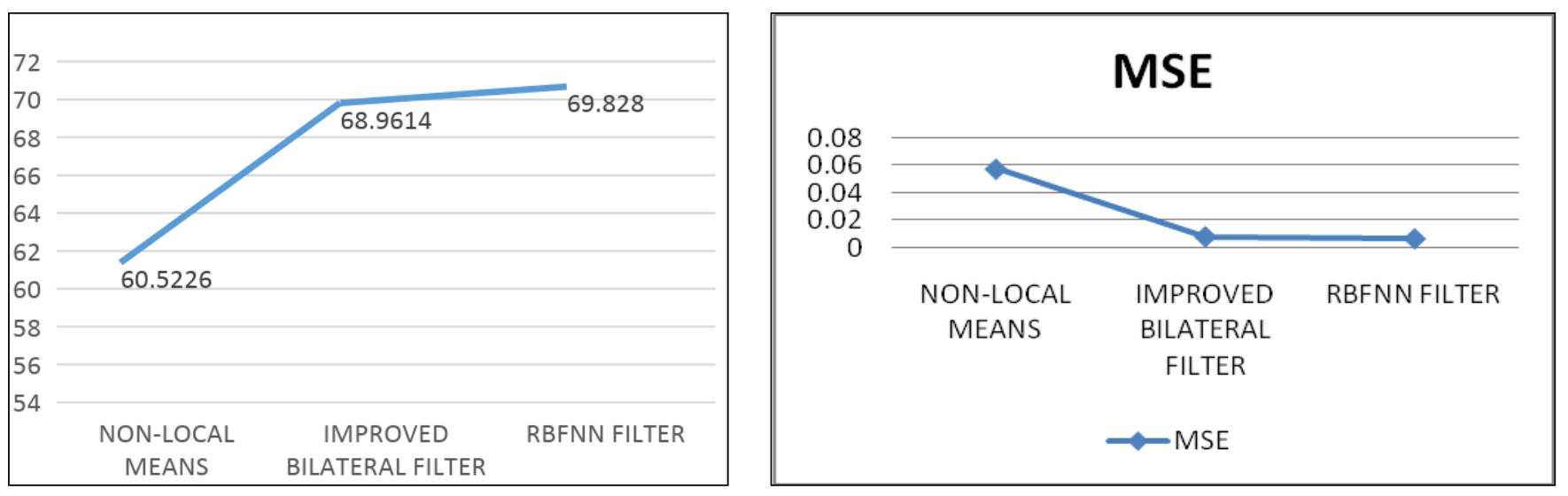

Figure 4 PSNR AND MSE Measure In Existing And Proposed Method

\section{REFERENCES}

1. Aleksandra Pizurica, Wilfred Philips, IgnaceLemahieu and Marc Acheory , (2003)"A Versatile Wavelet Domain Noise Filtration Technique for Medical Imaging “, IEEE Trans. On Medical Imaging, Vol. 22, No. 3

2. Ali Ranjbaran, Anwar Hasni Abu Hassan, MahboobeJafarpour and BaharRanjbaran, (2015)"ALaplacian based image filtering using switching noise Detector", Ranjbaran et al. Springer Plus DOI 10.1186/s40064-015-0846-5

3. Antoine Leory, PirreMozer, YohanPayan and JocelyneTroccaz, (2004) "Rigid Registration of Freehand 3D Ultrasound and CT-scan images of the Kidney", springer-Verlag Berlin Heidelberg

4. Donghwan Kim, SathishRamani and Jeffrey A. Fessler, (2015) "CombiningOrdered Subsets and Accelerated XRay CT Image Reconstruction”, IEEE Transactions on Medical Imaging, vol. 34, no. 1.

5. Daw-Tung Lin, (2006) "Computer-Aided Kidney Segmentation on Abdominal CT images", IEEE Trans. On Information Technology in Biomedicine, Vol. 10, No. 1

6. HilalNaimi, AmelBahaHouda Adamou-Mitiche, Lahce neMitiche, (2015) " Medical image denoising using dual tree complex thresholding wavelet transform and Wiener filter", Journal of King Saud University Computer and Information Sciences 27, 40-45.

7. HosseinTalebi, and PeymanMilanfar," Global Image Denoising, (2014) ", IEEE Transactions On Image Processing, VOL. 23, NO. 2, 755

8. ShashikantAgrawal and RajkumarSahu,, (2012) "Wavelet Based MRI Image Denoising Using Thresholding Techniques", IJSETR, Vol.1, pp.32-35

9. H.S. Bhadauria, M.L. Dewal ,(2013),"Medical image denoising using adaptive fusion of curvelet transform and total variation", Computers and Electrical Engineering 39 1451-1460

10. M.Kiddo,Y.Hirata.S.Yamada, K.Kondo,(2016) "Automatic Restoration of X-ray Photography by Estimating Point Spread Function", ISCIT ,pp. 1180-1184

11. Baron Sam B, Monisha IR, NithiyaDhevi K, (2016) "Denoising Technique of CT, MRI Abdominal Images Using Block Matching and Hybrid Filter" Research Journal of Pharmaceutical, Biological and Chemical Sciences,ISSN: 0975-8585, vol7(3) 1397.

\section{BIOGRAPHIES OF AUTHORS}

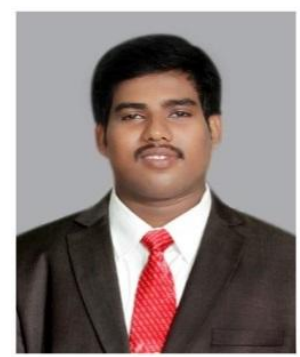

B. Baron Sam Currently he is an Research Scholar in the Department Computer Science and Engineering at Sathyabama Institute of science and Technology, Jeppiaar Nagar, Chennai, Tamil Nadu 600119

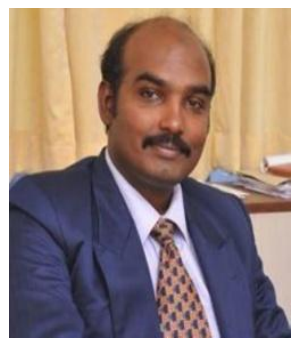

A. Lenin Fred Currently he is an principal at Mar Ephraem College of Engineering \& Technology, Malankara Hills, Elavuvilai, Marthandam, Tamil Nadu 629171.He received his B.E in Computer Science \& Engineering from Madurai Kamaraj University in 1995, M.E in Computer Science \& Engineering from Madurai Kamarajar University in 2001, Doctoral Degree in Computer Science \& Engineering, specialized in Digital Image Processing from ManonmaniamSundaranar University, Tirunelveli, India. 